\title{
Riesz MV-algebras and their logic
}

\author{
Antonio Di Nola ${ }^{1}$ Ioana Leuştean ${ }^{2}$ \\ ${ }^{1}$ Universita di Salerno, Via Ponte don Melillo, I84084 Fisciano, Salerno, Italy \\ ${ }^{2}$ University of Bucharest, Academiei 14, C.P. 010014, Bucharest, Romania
}

\begin{abstract}
We develop the general theory of RMV-algebras, which are essentially unit intervals in Riesz spaces with strong unit. Since the variety of RMV-algebras is generated by $[0,1]$, we get an equational characterization of the real product on $[0,1]$ interpreted as scalar multiplication.
\end{abstract}

Keywords: RMV-algebra, MV-algebra, Riesz space, Łukasiewicz logic.

\section{Introduction}

An $M V$-algebra is a structure $\left(A, \oplus,{ }^{*}, 0\right)$, where $(A, \oplus, 0)$ is an abelian monoid and the following identities hold for all $x, y \in A$ : $\left(x^{*}\right)^{*}=x, 0^{*} \oplus x=$ $0^{*}$ and $\left(x^{*} \oplus y\right)^{*} \oplus y=\left(y^{*} \oplus x\right)^{*} \oplus x$. Note that every MV-algebra $A$ is a bounded distributive lattice, where $x \vee y=x \oplus\left(x \oplus y^{*}\right)^{*}$ and $x \wedge y=\left(x^{*} \vee y^{*}\right)^{*}$ for any $x, y \in A$. If we set $x \odot y=\left(x^{*} \oplus y^{*}\right)^{*}$ then $\odot$ is the Eukasiewicz t-norm on $[0,1]$. The residuum is defined by $x \rightarrow y:=x^{*} \oplus y$.

MV-algebras are the algebraic structures of Łukasiewicz $\infty$-valued logic. The real unit interval $[0,1]$ equipped with the operations $x^{*}=1-x$ and $x \oplus y=\min (1, x+y)$ is the standard MV-algebra, i.e. an equation holds in any MV-algebra if and only if it holds in [0,1]. In [19] Mundici proved that MValgebras are categorically equivalent with abelian lattice-ordered groups with strong unit. Consequently, any MV-algebra is, up to isomorphism, the unit interval of an abelian lattice-ordered group with strong unit. We refer to [2] for all the unexplained notions concerning MV-algebras.

If we consider Riesz spaces [3, 16] with strong unit instead of lattice-ordered groups, then the unit interval is closed to the scalar multiplication with scalars from $[0,1]$.

The idea of considering these structures is also related to the problem of axiomatizing the real product on $[0,1]$. These investigations led to the definition of $P M V$-algebras (product $M V$-algebras) [4]. The analogue of Mundici's theorem for PMValgebras was obtained by Di Nola and Dvurečenskij [4]: there exists a categorical equivalence between PMV-algebras and lattice-ordered rings with strong unit ( $\ell$-rings). Due to a result of Isbell [12], the class of PMV-algebras is larger than intended. In [17], Montagna axiomatized the quasi-variety of PMV-algebras generated by $[0,1]$.
Interpreting the product as scalar multiplication with scalars from $[0,1]$, the standard algebra $[0,1]$ generates the variety of Riesz $M V$-algebras (RMValgebras, shortly). These structures are, up to isomorphism, unit intervals in Riesz spaces with strong unit. Our goal is to develop a theory for these structures and to investigate their relevance within MValgebras.

Note that RMV-algebras are particular MVmodules, structures defined in [6]. Consequently, some results presented in this paper are obtained from general results proved in $[6,14]$.

The second section contains basic facts on RMValgebras. We specialize Mundici's equivalence to RMV-algebras and Riesz spaces with strong unit and we establish an adjunction between MValgebras and RMV-algebras. Consequently, any MV-algebra has an RMV-algebra cover. We get a particular characterization for semisimple RMValgebra and we recall a construction from [5], where the Riesz spaces are proved to be categorical equivalent with a particular class of RMV-algebras.

In the third section we characterize the variety of $\mathrm{RMV}$-algebras and we prove that it is generated by the standard RMV-algebra [0,1]. We also characterize the free RMV-algebras with $n$ generators as algebras of Mc Naughton functions with real coefficients.

The last section present $\mathcal{L}_{R}$, a propositional calculus which has RMV-algebras as models. This calculus has standard completeness with respect to $[0,1]$. In the end we prove a theorem of approximation: any continuous function $h:[0,1]^{n} \rightarrow[0,1]$ is uniform limit of functions corresponding to formulas of $\mathcal{L}_{R}$.

\section{RMV-algebras}

In [6] the authors defined the structure of $M V$ module over a PMV-algebra. The RMV-algebras are the unital $\mathrm{MV}$-modules over $[0,1]$, where the PMV-algebra structure of $[0,1]$ is given by the real product. Hence the general theory of MV-modules $[6,14]$ can be applied to RMV-algebras.

Definition 2.1 A Riesz $M V$-algebra (RMValgebra) is a structure $(R, \cdot)$, where $R$ is an MV-algebra and $\cdot:[0,1] \times R \longrightarrow R$ is such that the following properties hold for any $x, y \in R$ and $r, q \in[0,1]$ :

$(\mathrm{RMV} 1)(r \cdot x) \odot(r \cdot y)=0$ and $r \cdot(x \oplus y)=(r \cdot x) \oplus(r \cdot y)$ whenever $x \odot y=0$, 
$(\operatorname{RMV} 2)(r \cdot x) \odot(q \cdot x)=0$ and $(r \oplus q) \cdot x=(r \cdot x) \oplus(q \cdot x)$ whenever $r \odot q=0$,

$(\operatorname{RMV} 3)(r \cdot q) \cdot x=r \cdot(q \cdot x)$,

$(\mathrm{RMV} 4) 1 \cdot x=x$.

We shall frequently denote an RMV-algebra $(R, \cdot)$ by its MV-algebra support $R$ and we shall simply write $r x$ instead of $r \cdot x$ for $r \in[0,1]$ and $x \in R$.

Example $2.2(1)([0,1], \cdot)$ is an RMV-algebra, where - is the real product. Moreover, due to a result of Hion [9][Chapter IV, Proposition 2], one can prove that this is the only structure of RMValgebra on $[0,1]$ and it will be called the standard $R M V$-algebra.

(2) If $X$ is a compact Hausdorff space, then $C(X)=\{f: X \rightarrow[0,1] \mid f$ continuous $\}$ is an RMValgebra, with the scalar multiplications defined by $(r f)(x):=r f(x)$ for any $x \in X$.

The following example is the motivation of our theory.

Example 2.3 The unit interval of a Riesz space. Let $(V, u)$ be a Riesz space with strong unit [16, 3]. Hence the unit interval $\Gamma(V, u)=\left([0, u]_{V}, \oplus,{ }^{*}, 0\right)$ is an MV-algebra by Mundici's categorical equivalence [18]. Moreover, $r x \in[0, u]$ whenever $r \in[0,1]$ and $x \in[0, u]$. It is straightforward that $\Gamma(V, u)$ is an RMV-algebra.

If $R$ is an RMV-algebra and $I \subseteq R$ is an MVideal, then $r x \in I$ for any $r \in[0,1]$ and $x \in I$ [6][Remark 3.15]. Hence, the MV-ideals and the RMV-ideals coincide, i.e. the MV-algebra congruences are compatible with the scalar multiplication. Consequently, if $f: R_{1} \rightarrow R_{2}$ is an MV-algebra homomorphism then $f(r x)=r f(x) r \in[0,1]$ and $x \in R_{1}$, so RMV-algebra homomorphisms are MValgebra homomorphisms between RMV-algebras, so we specialize Mundici's categorical equivalence as follows.

Theorem 2.4 [6] The category of RMV-algebras with MV-algebra homomorphisms is equivalent to the category of Riesz spaces with strong unit with unit preserving Riesz homomorphisms. As a consequence, for any RMV-algebra $R$ there exists a Riesz space with strong unit $(V, u)$ such that $R$ is isomorphic with $\Gamma(V, u)$.

Chang's representation theorem for MV-algebras [2] immediately yields a similar representation for RMV-algebras.

Theorem 2.5 Any RMV-algebra $R$ is isomorphic with a subdirect product of linearly ordered RMValgebras.

Proof. There is an MV-algebra embedding $h$ : $R \rightarrow \prod_{P \in \operatorname{Spec}(R)} R / P$, where $\operatorname{Spec}(R)$ is the prime ideal space of $R$. But any ideal $P$ is an RMV-algebra ideal, so $R / P$ is an RMV-algebra. Hence $h$ is an RMV-algebra embedding.

The relation between MV-algebras and RMValgebras can be expressed using the tensor product of MV-algebras $\otimes$ defined in [20].

Proposition 2.6 For any MV-algebra $A$, the tensor product $[0,1] \otimes A$ has an RMV-algebra structure such that the following properties hold:

(a) $r(q \otimes x)=(r q) \otimes x$ for any $r, q \in[0,1], x \in A$,

(b)the function $\iota_{A}: A \rightarrow[0,1] \otimes A$ defined by $\iota_{A}(x):=1 \otimes x$ for any $x \in A$ is an MV-algebra embedding.

Moreover, for any RMV-algebra $R$ and any MValgebra homomorphism $f: A \rightarrow R$ there exists a unique RMV-algebra homomorphism $f_{\otimes}:[0,1] \otimes A \rightarrow R$ such that $f_{\otimes} \circ \iota_{A}=f$.

Proof. (a) is proved in [7][Theorem 4.1].

(b) By [20][Proposition 2.3], $\iota_{A}$ is an MV-algebra homomorphism. The fact that $\iota_{A}$ is an embedding was proved by F. Montagna and T. Flaminio (private communication).

The above results asserts that any MV-algebra has an RMV-algebra hull. This construction yields an adjunction between the category $\mathcal{M V}$ of $\mathrm{MV}$ algebras and the category $\mathcal{R} \mathcal{M V}$ of RMV-algebras. Let us define the functors

$$
\mathcal{U}: \mathcal{R} \mathcal{M V} \rightarrow \mathcal{M V} \text { and } \mathcal{T}_{\otimes}: \mathcal{M V} \rightarrow \mathcal{R} \mathcal{M V}
$$

as follows: $\mathcal{U}$ is the forgetful functor forgets the scalar multiplication and $\mathcal{T}_{\otimes}(A):=[0,1] \otimes A$ for any MV-algebra $A$. If $h: A \rightarrow B$ is an MV-algebra homomorphism then $\iota_{B} \circ h: A \rightarrow[0,1] \otimes B$, using Proposition 2.6, we get an RMV-algebra homomorphism $\left(\iota_{B} \circ h\right)_{\otimes}:[0,1] \otimes B \rightarrow[0,1] \otimes A$. Hence we define $\mathcal{T}_{\otimes}(h):=\left(\iota_{B} \circ h\right)_{\otimes}$ whenever $h: A \rightarrow B$ is an MV-algebra homomorphism.

Theorem 2.7 $\left(\mathcal{T}_{\otimes}, \mathcal{U}\right)$ is an adjoint pair.

Proof. For a detailed proof in the general setting of MV-modules see [14][Proposition 7.29]. It is obvious that $\mathcal{T}_{\otimes}$ is a functor. Let $A$ be an MV-algebra and $R$ an RMV-algebra. By Proposition 2.6, for any MV-algebra homomorphism $f: A \rightarrow \mathcal{U}(R)$ there exists a unique RMV-algebra homomorphism $f_{\otimes}: \mathcal{T}_{\otimes}(A) \rightarrow R$ such that $\mathcal{U}\left(f_{\otimes}\right) \circ \iota_{A}=f$. This proves that $\mathcal{T}_{\otimes}$ is left adjoint to $\mathcal{U}$.

Proposition 2.8 An MV-algebra $A$ admits an RMV-algebra structure if and only if $A \simeq[0,1] \otimes A$.

Proof. If $A$ is an RMV-algebra then, by Proposition 2.6, there exists a unique RMV-algebra homomorphism $\left(I_{A}\right)_{\otimes}$ such that $\left(I_{A}\right)_{\otimes} \circ \iota_{A}=I_{A}$, where $I_{A}: A \rightarrow A$ is the identity function. We only have to prove that $\iota_{A} \circ\left(I_{A}\right)_{\otimes}=I_{[0,1] \otimes A}$, but this is true since the two functions coincide on the generators of 
$[0,1] \otimes A$, i.e. $r \otimes x=r(1 \otimes x)=r \iota_{A}(x)=\iota_{A}(r x)=$ $\iota_{A}\left(\left(I_{A}\right)_{\otimes}(r \otimes x)\right)$ for any $r \in[0,1]$ and $x \in A$.

We further emphasize some properties of RMValgebras.

Lemma 2.9 For any RMV-algebra $(R, \cdot)$ the following properties hold.

(a) The function $r \mapsto r \cdot 1_{R}$ is an embedding of $[0,1]$ in $R$.

(b) For any maximal ideal $M \subseteq R, R / M \simeq[0,1]$.

Proof. (a) By Theorem 2.4, we can take $R=$ $\Gamma(V, u)$ for some Riesz space with strong unit $u$ and the intended result follows from the properties of Riesz spaces.

(b) For any maximal ideal $M \subseteq R$, the MV-algebra $R / M$ is simple, so it is a subalgebra of $[0,1]$. Using (a), we get $R / M \simeq[0,1]$.

As a consequence of the previous lemma, the only simple RMV-algebra is $[0,1]$.

Recall that an MV-algebra is archimedean if the corresponding lattice-ordered group is archimedean. Archimedean MV-algebras are equivalent with the semisimple ones, i.e. those with the property that $\operatorname{Rad}(A)=\bigcap\{M \subseteq A \mid M \in \operatorname{Max}(A)\}=\{0\}$, where $\operatorname{Max}(A)$ is the maximal ideal space of $A$. Consequently, semisimple and archimedean RMValgebras will coincide. Since the unique simple RMV-algebra is [0,1], any semisimple RMV-algebra is isomorphic with a subdirect product of copies of $[0,1]$.

For any MV-algebra $A, \operatorname{Max}(A)$ is a compact Hausdorff space with respect to the spectral topology. For a nonempty set $X$, an MV-subalgebra $S$ of $[0,1]^{X}$ is separating if whenever $x \neq y \in X$ there exists $f \in S$ such that $f(x)=0$ and $f(y)>0$. It is known that any archimedean MV-algebra $A$ is isomorphic with a separating $\mathrm{MV}$-subalgebra of $C(\operatorname{Max}(A))[2]$.

We can further specialize the characterization of semisimple RMV-algebras.

Let us firstly recall the MV-algebraic version of Stone-Weierstrass theorem.

Theorem 2.10 (Stone-Weierstrass for $R M V$ algebras )[15] Assume $X$ is a compact Hausdorff space. Every separating RMV-subalgebra $A$ of $C(X)$ is dense in $C(X)$ with respect to the sup-norm.

Theorem 2.11 (Characterization of semisimple $R M V$-algebra) Any semisimple RMV-algebra $R$ is isomorphic with a dense (w.r.t. to the sup-norm) subalgebra of $C(\operatorname{Max}(R))$.

Proof. By Stone-Weierstrass theorem, $R$ is dense in $C(X)$.

In the end of this section we recall an important construction defined in [5]
Remark 2.12 Let $V$ be a Riesz space and $\mathbb{R} \times_{\text {lex }}$ $V$ be the lexicographic product. Hence $(1,0)$ is a strong unit, so $R=\Gamma\left(\mathbb{R} \times_{\text {lex }} V,(1,0)\right)$ is an $\mathrm{RMV}$-algebra. Denote $\mathcal{R} \mathcal{M} \mathcal{V}_{\text {lex }}$ the class of RMValgebras $R$ with the property that $R \simeq \Gamma\left(\mathbb{R} \times{ }_{\text {lex }}\right.$ $V,(1,0))$ for some Riesz space $V$. This class is axiomatized in [5].

Theorem 2.13 [5][Theorem 4.6] $\mathcal{R} \mathcal{M} \mathcal{V}_{\text {lex }}$ is equivalent with the category of Riesz spaces.

If $T$ is an MV-algebra and $a \neq 0$ in $T$ then the interval $\left([0, a], \oplus_{a},{ }^{* a}, 0\right)$ is an MV-algebra with the operations defined by: $x \oplus_{a} y:=(x \oplus y) \wedge a, x^{* a}:=$ $x^{*} \odot a$. Note that, whenever $T$ is an RMV-algebra, the interval $[0, a]$ is closed to scalar multiplication, so $[0, a]$ is an RMV-algebra.

The following result allows us to assert that the class $\mathcal{R} \mathcal{M} \mathcal{V}_{\text {lex }}$ stands to RMV-algebras as perfect $\mathrm{MV}$-algebras stand to MV-algebras.

Lemma 2.14 For any RMV-algebra $R$ there exists an RMV-algebra $T$ in $\mathcal{R} \mathcal{M} \mathcal{V}_{\text {lex }}$ and an element $a \neq$ 0 in $T$ such that $R \simeq[0, a]$.

Proof. If $R$ is an RMV-algebra then $R \simeq \Gamma(V, u)$ for some Riesz space with strong unit $(V, u)$. Set $T:=\Gamma\left(\mathbb{R} \times_{\text {lex }} V,(1,0)\right)$ and $a:=(0, u)$. Then the intended conclusion is straightforward.

\section{Equational characterization. Free RMV-algebras.}

We show that the class of RMV-algebras is the variety generated by $[0,1]$.

Theorem 3.1 If $R$ is an MV-algebra and : : $[0,1] \times R \rightarrow R$ then $(R, \cdot)$ is an RMV-algebra if and only if the following identities are satisfied for any $r, q \in[0,1]$ and $x, y \in R$ :

$(\mathrm{R} 1)(r x) \odot((r \vee q) x)^{*}=0$,

(R2) $\left(r \odot q^{*}\right) x=(r x) \odot((r \wedge q) x)^{*}$,

(R3) $r(q x)=(r \cdot q) x$

(R4) $r\left(x \odot y^{*}\right)=(r x) \odot(r y)^{*}$,

(R5) $1 x=x$.

Proof. It follows by [8][Corollary 3.13]. For a detailed proof one can see [14][Corollary 6.45].

The free objects in a variety always exists. In the category of RMV-algebras we get the following particular characterization.

Proposition 3.2 For any set $X$, the free RMValgebra generated by $X$ is $[0,1] \otimes \operatorname{Free}_{M V}(X)$, where $\operatorname{Free}_{M V}(X)$ is the free MV-algebra generated by $X$.

Proof. It is straightforward by Proposition 2.6.

We prove that an identity holds in the theory of RMV-algebras if and only if it holds in the 
standard RMV-algebra [0,1]. Our approach follows closely the proof of Chang's completeness theorem for Łukasiewicz logic [1]. To any sentence in the first-order theory of RMV-algebras we associate a sentence in the first-order theory of Riesz spaces such that the satisfiability is preserved by the $\Gamma$ functor. The first-order theory of RMValgebras, as well as the theory of Riesz spaces, are obtained considering for each scalar $r$ an unary function $\rho_{r}$ which denotes in a particular model the scalar multiplication by $r$, i.e. $x \stackrel{\rho_{r}}{\mapsto} r x$. In the following, the language of RMV-algebras is $\mathcal{L}_{R M V}=\left\{\oplus,{ }^{*}, 0,\left\{\rho_{r}\right\}_{r \in[0,1]}\right\}$ and the language of Riesz spaces is $\mathcal{L}_{\text {Riesz }}=\left\{\leq,+,-, \vee, \wedge, 0,\left\{\rho_{r}\right\}_{r \in \mathbb{R}}\right\}$.

Let $t\left(v_{1}, \ldots, v_{k}\right)$ be a term of $\mathcal{L}_{R M V}$ and $v$ a propositional variable different from $v_{1}, \ldots, v_{k}$. We define $\widetilde{t}$ as follows:

- if $t=0$ then $\widetilde{0}$ is 0 ,

- if $t=v$ then $\tilde{t}$ is $v$

- if $t=t_{1}^{*}$ then $\tilde{t}$ is $v-\widetilde{t_{1}}$,

- if $t=t_{1} \oplus t_{2}$ then $\tilde{t}$ is $\left(t_{1}+t_{2}\right) \wedge v$,

- if $t=\rho_{r}\left(t_{1}\right)$ then $\tilde{t}$ is $\rho_{r}\left(\widetilde{t_{1}}\right)$.

Let $\varphi\left(v_{1}, \ldots, v_{k}\right)$ be a formula of $\mathcal{L}_{R M V}$ such that all the free and bound variables of $\varphi$ are in $\left\{v_{1}, \ldots, v_{k}\right\}$ and $v$ a propositional variable different from $v_{1}, \ldots$, $v_{k}$. We define $\widetilde{\varphi}$ as follows:

- if $\varphi$ is $t_{1}=t_{2}$ then $\widetilde{\varphi}$ is $\widetilde{t_{1}}=\widetilde{t_{2}}$,

- if $\varphi$ is $\neg \psi$ then $\widetilde{\varphi}$ is $\neg \widetilde{\psi}$,

- if $\varphi$ is $\psi \vee \chi$ then $\tilde{\varphi}$ is $\widetilde{\psi} \vee \widetilde{\chi}$ and similarly for

$\wedge, \rightarrow, \leftrightarrow$

- if $\varphi$ is $\left(\forall v_{i}\right) \psi$ then $\widetilde{\varphi}$ is

$\forall v_{i}\left(\left(0 \leq v_{i}\right) \wedge\left(v_{i} \leq v\right) \rightarrow \widetilde{\psi}\right)$,

- if $\varphi$ is $\exists v_{i} \psi$ then $\widetilde{\varphi}$ is

$\exists v_{i}\left(\left(0 \leq v_{i}\right) \wedge\left(v_{i} \leq v\right) \rightarrow \widetilde{\psi}\right)$.

Thus to any formula $\varphi\left(v_{1}, \ldots, v_{k}\right)$ of $\mathcal{L}_{R M V}$ we associate a formula $\widetilde{\varphi}\left(v_{1}, \ldots, v_{k}, v\right)$ of $\mathcal{L}_{\text {Riesz }}$. As a consequence, to any sentence $\sigma$ of $\mathcal{L}_{R M V}$ corresponds a formula with only one free variable $\widetilde{\sigma}(v)$ of $\mathcal{L}_{\text {Riesz }}$.

Proposition 3.3 Let $(V, u)$ be a Riesz space with strong unit and $R=\Gamma(V, u)$. If $\sigma$ is a sentence in the first-order theory of RMV-algebras then

$$
R \models \sigma \text { if and only if } V \models \widetilde{\sigma}[u] .
$$

Proof. By structural induction on terms it follows that $t\left[a_{1}, \ldots, a_{n}\right]=\widetilde{t}\left[a_{1}, \ldots, a_{n}, u\right]$ whenever $t\left(v_{1}, \ldots, v_{n}\right)$ is a term of $\mathcal{L}_{R M V}$ and $a_{1}, \ldots, a_{n} \in R$. The rest of the proof is straightforward.

Theorem 3.4 An equation $\sigma$ in the theory of RMV-algebras holds in all RMV-algebras if and only if it holds in the standard RMV-algebra $[0,1]$.

Proof. One implication is obvious. To prove the other one, let $R$ be an RMV-algebra such that $R \not \forall \sigma$. Since $R \simeq \Gamma(V, u)$ for some Riesz space with strong unit $(V, u)$, we have that $\Gamma(V, u) \not \forall \sigma$. Using Proposition 3.3, we infer that $V \not \models \widetilde{\sigma}[u]$ in the theory of Riesz spaces. Since the order relation in any lattice can be expressed equationally, we note that $\widetilde{\sigma}(v)$ is a quasi-identity. By [13][Corollary 2.6] a quasi-identity is satisfied by all Riesz spaces if and only if it is satisfied by $\mathbb{R}$. Hence there exists a real number $c \geq 0$ such that $\mathbb{R} \forall \widetilde{\sigma}[c]$. Since $\mathbb{R} \models \widetilde{\sigma}[0]$, we get $c>0$. If follows that $f: \mathbb{R} \rightarrow \mathbb{R}$ defined by $f(x) \mapsto x / c$ is an automorphism of Riesz spaces. We infer that $\mathbb{R} \not \forall \widetilde{\sigma}[1]$, so $[0,1] \not \models \sigma$.

Corollary $3.5[0,1]$ generates the variety of RMValgebras.

Given $t\left(v_{1}, \cdots, v_{n}\right)$ a term in $\mathcal{L}_{R M V}$ we define the term function $f_{t}:[0,1]^{n} \rightarrow[0,1]$ as usual (see [?] for the general theory). In the theory of MV-algebras, the term functions are Mc Naughton functions [2], i.e. continuous piecewise affine functions with integer coefficients. We immediately obtain a similar description for the term functions in $\mathcal{L}_{R M V}$.

Definition 3.6 Let $n>1$ be a natural number. A Mc Naughton function with real coefficients is a continuous function $f:[0,1]^{n} \longrightarrow[0,1]$ which satisfies the following condition:

there exists a finite number of affine functions (with real coefficients) $q_{1}, \ldots, q_{k}: \Re^{n} \longrightarrow \Re$ such that for any $\left(a_{1}, \ldots, a_{n}\right) \in[0,1]^{n}$ there is $i \in\{1, \ldots, k\}$ with $f\left(a_{1}, \ldots, a_{n}\right)=q_{i}\left(a_{1}, \ldots, a_{n}\right)$.

Theorem 3.7 If $t\left(v_{1}, \cdots, v_{n}\right)$ is a term in $\mathcal{L}_{R M V}$ then the term function $f_{t}:[0,1]^{n} \rightarrow[0,1]$ is a Mc Naughton function with real coefficients.

Proof. We prove the conclusion by structural induction on terms:

- if $t$ is $v_{i}$ for some $i \in\{1, \ldots, n\}$ then $f_{t}=\pi_{i}$ (the $i$-th projection);

- if $t$ is $t_{1} \oplus t_{2}$ and let $q_{1}, \ldots, q_{m}$ be the polynomials of $f_{t_{1}}$ and $p_{1}, \ldots, p_{k}$ be the polynomials of $f_{t_{2}}$; then $f_{t}$ is defined by the polynomials $\{1\} \cup\left\{s_{i j}\right\}_{i, j}$, where $s_{i j}=q_{i}+p_{j}$ for any $i \in\{1, \ldots, s\}$ and $j \in\{1, \ldots, r\}$;

- $t$ is $t_{1}^{*}$ and $q_{1}, \ldots, q_{s}$ are the corresponding polynomials of $f_{t_{1}}$, then $\mathrm{i} 1-q_{1}, \ldots, 1-q_{s}$ are the polynomials of $f_{t}$;

- $t$ is $\rho_{r}\left(t_{1}\right)$ for some $r \in[0,1]$ and $q_{1}, \ldots, q_{s}$ are the corresponding polynomials of $f_{t_{1}}$, then i $r q_{1}, \ldots, r q_{s}$ are the polynomials of $f_{t}$.

Remark 3.8 Term functions and Mc Naughton functions with integer coefficients coincide in the case of MV-algebras. It is an open question if this holds for RMV-algebras too:

(*) given $f:[0,1]^{n} \rightarrow[0,1]$ a Mc Naughton function with real coefficients, can we find a term $t$ in $\mathcal{L}_{R}$ such that $f=f_{t}$ ?

Note that, for $f:[0,1]^{n} \rightarrow[0,1]$ a Mc Naughton function with real coefficients, there are finite sets $I$ and $J$ such that

$$
f=\bigvee_{i \in I} \bigwedge_{j \in J} f_{i j}
$$


where $f_{i j}:[0,1]^{n} \rightarrow \mathbb{R}$ are affine functions with real coefficients [21, Theorem 2.1]. It follows that it would be enough to answer $(\star)$ for affine functions with real coefficents.

In Section 4 we develop a propositional calculus for RMV-algebras. Since the primary connectives of Eukasiewicz logic are $\rightarrow$ and $\neg$, we have to provide an equational characterization of the scalar multiplication using implication and negation.

Remark 3.9 [14, Section 6.4] Let $R$ be an MValgebra and $\circ:[0,1] \times R \rightarrow R$ such that the following properties hold for any $x, y \in R$ and $r, q \in[0,1]$ :

$\left(1^{\circ}\right) r \circ(x \rightarrow y)=(r \circ x) \rightarrow(r \circ y)$,

$\left(2^{\circ}\right)\left(r \odot q^{*}\right) \circ x=((r \wedge q) \circ x) \rightarrow(r \circ x)$,

$\left(3^{\circ}\right) r \circ(q \circ x)=(r \cdot q) \circ x$,

$\left(4^{\circ}\right)((r \vee q) \circ x) \rightarrow(r \circ x)=1$,

$\left(5^{\circ}\right) 1 \circ x=x$.

We call dual $R M V$-algebra a structure $(R, \circ)$ as above. If $R$ is an MV-algebra and $\circ:[0,1] \times R \rightarrow R$ we define

$r \cdot x:=\left(r \circ\left(x^{*}\right)\right)^{*}$ for any $x \in R, r \in[0,1]$.

Hence $(R, \cdot)$ is an RMV-algebra if and only if $(R, \circ)$ is a dual RMV-algebra [14].

\section{A propositional calculus for RMV-algebra}

We develop in a classical way a propositional calculus $\mathcal{L}_{R}$ that have RMV-algebra as models. One can see [14] for detailed proof in the general setting of MV-modules. Note that Theorem 4.5 and Theorem 4.6 are not proved in general.

The language of the propositional calculus $\mathcal{L}_{R}$ consists of:

- denumerable many propositional variables: $v_{1}$, $\ldots, v_{n}, \ldots$

(the set of all the propositional variables will be denoted by $V a r)$,

- the logical connectives of $\mathcal{L}: \rightarrow$ (binary) and $\neg$ (unary),

- unary logical connectives : $\nabla_{r}$ for any $r \in[0,1]$, parentheses: ( and ).

We denote by $\operatorname{Form}\left(\mathcal{L}_{R}\right)$ the set of formulas, which are defined inductively as usual.

Definition 4.1 The axioms of $\mathcal{L}_{R}$ are defined as follows:

a formula which has one of the following forms is an axiom (where $\varphi, \psi$ and $\chi$ are arbitrary formulas and $r, q$ are arbitrary elements of $[0,1])$ :

(L1) $\varphi \rightarrow(\psi \rightarrow \varphi)$,

(L2) $(\varphi \rightarrow \psi) \rightarrow((\psi \rightarrow \chi) \rightarrow(\varphi \rightarrow \chi))$,

(L3) $((\varphi \rightarrow \psi) \rightarrow \psi) \rightarrow((\psi \rightarrow \varphi) \rightarrow \varphi)$,

(L4) $(\neg \psi \rightarrow \neg \varphi) \rightarrow(\varphi \rightarrow \psi)$

$(\mathrm{F} 1) \diamond_{r}(\varphi \rightarrow \psi) \leftrightarrow\left(\diamond_{r} \varphi \rightarrow \diamond_{r} \psi\right)$,

$(\mathrm{F} 2) \diamond_{\left(r \odot q^{*}\right)} \varphi \leftrightarrow\left(\nabla_{r \wedge q} \varphi \rightarrow \nabla_{r} \varphi\right)$,

(F3) $\diamond_{r} \diamond_{q} \varphi \leftrightarrow \nabla_{r \cdot q} \varphi$,

(F4) $\diamond_{r \vee q} \varphi \rightarrow \diamond_{r} \varphi$

(F5) $\diamond_{1} \varphi \leftrightarrow \varphi$.
Note that (L1)-(L4) are the axiom of Łukasiewicz logic. Note that the axioms (F1)-(F5) are the duals of (R1)-(R5) if we consider that the term function associated to $\nabla_{r} \varphi$ is $\left(r f_{\varphi}^{*}\right)^{*}$, where $f_{\varphi}$ is the term function of $\varphi$.

The deduction rule of $\mathcal{L}_{R}$ is modus ponens: $\frac{\varphi, \varphi \rightarrow \psi}{\psi}$. Proofs are defined as usual.

\section{Proposition 4.2 (Deduction theorem)}

$\Theta \cup\{\varphi\} \vdash \psi$ iff $\Theta \vdash \varphi^{n} \rightarrow \psi$ for some $n \geq 1$, where $\varphi^{n}$ denotes $\underbrace{\varphi \odot \cdots \odot \varphi}_{n \text { times }}$.

We define the Lindenbaum-Tarski algebra. In the sequel $\Theta \subseteq \operatorname{Form}\left(\mathcal{L}_{R}\right)$ is a fixed set of formulas. For any two formulas $\varphi$ and $\psi$ we define

$$
\varphi \equiv_{\Theta} \psi \text { iff } \Theta \vdash \varphi \rightarrow \psi \text { and } \Theta \vdash \psi \rightarrow \varphi \text {. }
$$

Since $\equiv_{\Theta}$ is an equivalence relation on $\operatorname{Form}\left(\mathcal{L}_{R}\right)$, we denote $[\varphi]_{\Theta}$ the equivalence class of $\varphi$ with respect to $\equiv_{\Theta}$.

On $\operatorname{Form}\left(\mathcal{L}_{R}\right) / \equiv_{\Theta}$ we define the following operations:

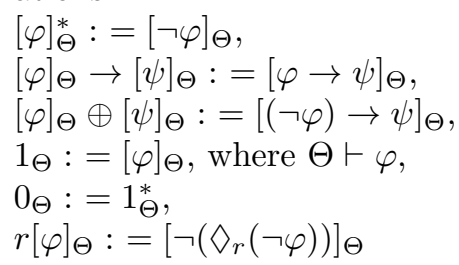

With the above operations, $\operatorname{Form}\left(\mathcal{L}_{R}\right) / \equiv_{\Theta}$ is an RMV-algebra by Remark 3.9. When $\Theta=\emptyset$, we denote $\operatorname{Lind} a_{R}:=\operatorname{Form}\left(\mathcal{L}_{R}\right) / \equiv \emptyset$ and this is the Lindenbaum-Tarski algebra of RMV-logic.

Since the Lindenbaum-Tarski algebra is freely generated by the set of variables, by Proposition 3.2 , we get the following.

Corollary 4.3 $\operatorname{Lind}_{R} \simeq[0,1] \otimes \operatorname{Lind}_{L u k}$, where Lind $a_{L u k}$ is the Lindenbaum-Tarski algebra of Łukasiewicz logic.

Let $R$ be an RMV-algebra. Following Remark 3.9, an R-evaluation is a function $e: \operatorname{Form}\left(\mathcal{L}_{R}\right) \rightarrow R$ which satisfies the following conditions:

(e1) $e(\varphi \rightarrow \psi)=e(\varphi)^{*} \oplus e(\psi)$,

(e2) $e(\neg \varphi)=e(\varphi)^{*}$,

(e3) $e\left(\nabla_{a} \varphi\right)=\left(a e(\varphi)^{*}\right)^{*}$,

for any $\varphi \in \operatorname{Form}\left(\mathcal{L}_{R}\right)$ and $r \in[0,1]$.

The notions of satisfaction and semantic consequence are defined as usual.

According to [11], the system $\mathcal{L}_{R}$ is a core fuzzy logic, hence the strong completeness with respect to linearly ordered structures follows by $[11$, Theorem 2.11].

For $\mathcal{L}_{R}$ we also prove completeness with respect to the standard model. 
Theorem 4.4 (Strong completeness theorem) Assume $\Theta$ be a set of formulas and $\varphi$ a formula of $\mathcal{L}_{R}$. The following are equivalent:
(a) $\Theta \vdash \varphi$,
(b) $\Theta \models{ }_{R} \varphi$ for any RMV-algebra $R$,
(c) $\Theta \models_{R} \varphi$ for any linearly-ordered RMV-algebra $R$.

Proof. The equivalence of (a) and (b) is straightforward. The equivalence with (c) follows by Theorem 2.5. See also [11, Theorem 2.11].

Theorem 4.5 (Standard completeness) For a formula $\varphi$ of $\mathcal{L}_{R}$, the following are equivalent:

(a) $\vdash \varphi$,
(b) $\models_{[0,1]} \varphi$.

Proof. It follows by Theorem 3.4.

As a direct consequence of the standard completeness it follows that the logic of RMV-algebras is a conservative extension of Łukasiewicz logic.

Finally, we prove an approximation result.

Theorem 4.6 (Approximation of continuous functions) Let $n \geq 1$ be a natural number. For any continuous function $h:[0,1]^{n} \rightarrow[0,1]$ there exists a sequence of formulas $\left(\varphi_{n}\right)_{n}$ of $\mathcal{L}_{R}$ such that $h$ is the uniform limit of $\left(f_{\varphi_{n}}\right)_{n}$.

Proof. If Form $_{n}$ is the set of the formulas which contain only the variables $v_{1}, \ldots, v_{n}$, then $R_{n}=$ Form $_{n} / \equiv_{\emptyset}$ is the free RMV-algebra with $n$ generators. By Theorem 3.7, $R_{n}$ is a semisimple RMV-algebra. By Theorem 2.11, $R_{n}$ is dense in $C(X)$ in the sup-norm which proves our result.

Remark 4.7 The logical system briefly presented in this chapter is strongly related with Rational Łukasiewicz Logic developed in [10], where only multiplication by rationals is considered. The algebraic structures of Rational Łukasiewicz Logic are the divisible MV-algebras. Our system is also a conservative extension of Rational Łukasiewicz Logic.

\section{Acknowledgment}

I. Leuştean was supported by the strategic grant POSDRU/89/1.5/S/58852, Project "Postdoctoral programme for training scientific researchers" cofinanced by the European Social Found within the Sectorial Operational Program Human Resources Development 2007-2013.

\section{References}

[1] C. C. Chang, A new proof of the completeness of the Eukasiewicz axioms, Transactions of the American Mathematical Society 93, pages 7480, 1959.

[2] R. Cignoli, I.M.L. D'Ottaviano, D. Mundici, Algebraic Foundations of many-valued Reasoning, Kluwer, Dordrecht, 2000.
[3] E. de Jonge, A.C.M. van Rooij, Introduction to Riesz spaces. Mathematical Centre Tracts 78, Amsterdam, 1977.

[4] A. Di Nola , A. Dvurečenskij, Product MValgebras, Multiple -Valued Logics 6, pages 193215, 2001.

[5] A. Di Nola, A. Lettieri, Coproduct MValgebras, nonstandard reals, and Riesz spaces, J. Algebra 185, No.3, pages 605-620, 1966.

[6] A. Di Nola, I. Leuştean, P. Flondor, MVmodules, Journal of Algebra 267, pages 21-40, 2003.

[7] P. Flondor, I. Leuştean, Tensor products of MV-algebras, Soft Computing 7, pages 446-457, 2003.

[8] P. Flondor, I. Leuştean, MV-algebras with operators (the commutative and the noncommutative case), Discrete Mathematics 274, pages 41-76, 2004.

[9] L. Fuchs, Partially Ordered Algebraic Systems, Pergamon Press, 1963.

[10] B. Gerla, Rational Łukasiewicz logic and DMV-algebras, Neural Networks World, vol 11, pages 579-584, 2001.

[11] P. Hájek, P. Cintula, On theories and models in fuzzy predicate logics, The Journal of Symbolic Logic 71, pages 863888, 2006.

[12] J.R. Isbell, Notes on ordered rings, Algebra Universalis, vol. 1, pages 393399, 1971.

[13] C.C.A. Labuschagne, C.J. van Alten, On the variety of Riesz spaces, Indagationes Mathematicae, N.S., 18 (1), pages 61-68, 2007.

[14] I. Leuştean, Contributions to the theory of MV-algebras: MV-modules, Editura Universitara, 2009. PhD Thesis, University of Bucharest, 2004.

[15] I. Leustean, V. Marra, Kakutani duality for MV-algebras, draft.

[16] W.A.J. Luxemburg, A.C. Zaanen, Riesz Spaces I, North-Holland, Amsterdam, 1971.

[17] F. Montagna, Subreducts of MV-algebras with product and product residuation, Algebra Universalis 53, pages 109-137, 2005.

[18] D. Mundici, Interpretation of AF $\mathrm{C}^{*}$-algebras in Łukasiewicz sentential calculus, J. Functional Analysis 65, pages 15-63, 1986.

[19] D. Mundici, Averaging the truth value Łukasiewicz logic, Studia Logica 55, pages 113127, 1995.

[20] D. Mundici, Tensor products and the LoomisSikorski theorem for MV-algebras, Advances in Applied Mathematics 22, pages 227-248, 1999.

[21] S. Ovchinnikov, Max-Min Representation of Piecewise Linear Functions, Beiträge zur Algebra und Geometrie Contributions to Algebra and Geometry Volume 43, No. 1, pages 297-302, 2002. 